\title{
Hyperfine Interactions and Spin Transport in Ferromagnet-Semiconductor Heterostructures
}

\author{
M. K. Chan, ${ }^{1}$ Q. O. Hu, ${ }^{2}$ J. Zhang, ${ }^{1}$ T. Kondo, ${ }^{1, *}$ C. J. Palmstrøm, ${ }^{2}$ and P. A. Crowell ${ }^{1}$ \\ ${ }^{1}$ School of Physics and Astronomy, \\ University of Minnesota, Minneapolis, MN 55455 \\ ${ }^{2}$ Departments of Electrical and Computer Engineering and Materials, \\ University of California, Santa Barbara, CA 93106
}

\begin{abstract}
Measurements and modeling of electron spin transport and dynamics are used to characterize hyperfine interactions in Fe/GaAs devices with $n$-GaAs channels. Ga and As nuclei are polarized by electrically injected electron spins, and the nuclear polarization is detected indirectly through the depolarization of electron spins in the hyperfine field. The dependence of the electron spin signal on injector bias and applied field direction is modeled by a coupled drift-diffusion equation, including effective fields from both the electronic and nuclear polarizations. This approach is used to determine the electron spin polarization independently of the assumptions made in standard transport measurements. The extreme sensitivity of the electron spin dynamics to the nuclear spin polarization also facilitates the electrical detection of nuclear magnetic resonance.
\end{abstract}


Recent observations of electrical spin injection and detection in ferromagnet - semiconductor (FS) devices have been confirmed by their demonstrated sensitivity to electron spin precession. $\underline{1}, 2,3,4,5,6$ There remain, however, several important issues which must be resolved in order to interpret spin transport measurements. For example, in n-GaAs doped near the metal-insulator transition, spin dynamics are influenced profoundly by hyperfine interactions..$\underline{-}$ Coupling between electron and nuclear spins leads to significant deviations from the expectations of simple drift-diffusion models. 1.5 .7 More importantly, the quantitative determination of the electron spin polarization in a FS device using standard transport measurements is based on assumptions about boundary conditions and densities of states that are not directly verifiable. A detailed understanding of hyperfine interactions could allow for an independent measurement of the electron spin polarization.

In this article we present measurements employing electrical generation and detection of dynamic nuclear polarization (DNP) in Fe/n-GaAs heterostructures. Non-equilibrium spin polarization is electrically injected into a GaAs channel, inducing DNP $\underline{\underline{8}}, \underline{9}, \underline{0}$ We show that the resulting dynamics of the combined electron-nuclear spin system can be described in a completely self-consistent manner. This approach allows for a measurement of the electron spin polarization that is independent of assumptions about either boundary conditions at the Fe/GaAs interface or the electronic densities of states in the ferromagnet or the semiconductor. Finally, we show that the spin-polarized electrons in GaAs in combination with the ferromagnetic detector can be used as a sensitive probe of nuclear magnetic resonance.

A schematic diagram of one of our devices is shown in Fig. 1(a) 1,2,11 The heterostructures consist of a $2.5 \mu \mathrm{m}$ thick Si-doped n-GaAs $\left(n=5 \times 10^{16} \mathrm{~cm}^{-3}\right)$ channel and $5 \mathrm{~nm}$ thick Fe electrodes that are deposited epitaxially on the GaAs (100) surface. A Schottky tunnel barrier is formed by a $n \rightarrow n^{+}\left(\sim 5 \times 10^{18} \mathrm{~cm}^{-3}\right)$ GaAs transition layer ${ }^{12}$ between the Fe and the $n$-type channel. The Fe injection and detection contacts are $7 \times 50 \mu \mathrm{m}^{2}$ and $4 \times 50 \mu \mathrm{m}^{2}$ respectively, with a center to center gap of $9.5 \mu \mathrm{m}$. DNP is observed in all heterostructures of this general design below $80 \mathrm{~K}$. At higher temperatures, spin-polarized electrons are no longer bound to the donor sites, thus suppressing the hyperfine coupling. $\frac{8}{}$ The discussion in this paper is based on data obtained at $T=60 \mathrm{~K}$.

Measurements are performed in the non-local ${ }^{2,13}$ or 3 -terminal $\frac{1,4}{\underline{4}}$ configurations shown in Fig. 1(a). Under forward bias, electrons tunnel from GaAs into the Fe injector (contact $b$ ). Majority spins aligned in the $x$ direction accumulate at the injector interface ${ }^{\underline{1}}$ and diffuse 


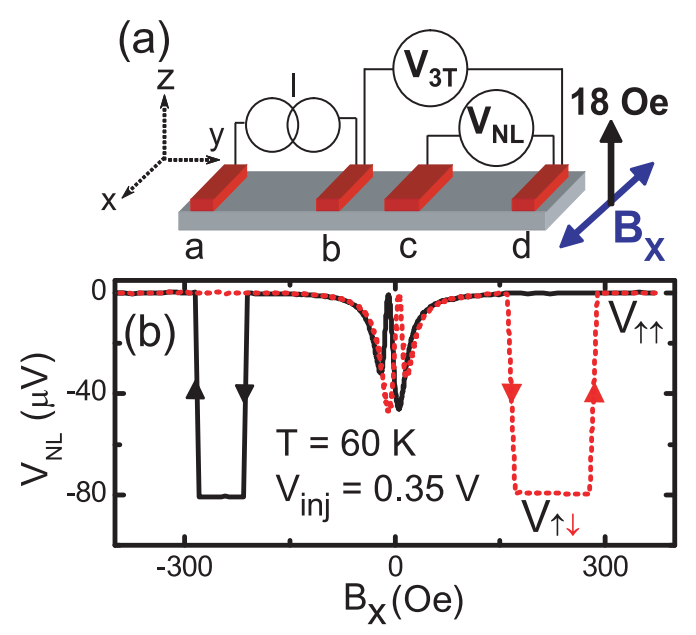

FIG. 1: (Color online) (a) Schematic of the lateral spin transport device. I establishes a forward bias at contact $b$. The spin polarization in the GaAs channel at contact $b$ or $c$ is determined by measuring the 3 -terminal voltage $V_{3 T}$ or non-local voltage $V_{N L}$ relative to reference contact $d$ (drawing not to scale). (b) $V_{N L}$ for longitudinal field $B_{x}$ swept from large positive field (black line) and from large negative field (red dotted line).

to the non-local detector (contact $c$ ). Contact $d$, located $290 \mu \mathrm{m}$ away, serves as a voltage reference. A longitudinal magnetic field $B_{x}$ is swept along the Fe easy axis ([110] direction, labeled $x$ in Fig. 1(a)) at a rate of $0.025 \mathrm{Oe} / \mathrm{s}$ in order to ensure that the nuclear spin polarization is in equilibrium. A small static transverse field of $B_{z}=18$ Oe is applied in the $z$ direction. This field does not perturb the magnetization of the contacts.

We first consider non-local spin-valve measurements. We observe jumps in the non-local voltage $V_{N L}$ when the injector and detector magnetizations switch between parallel and antiparallel states, as shown in Fig. 1(b) after subtraction of a spin-independent background. The magnitude of the voltage jump $V_{\uparrow \uparrow}-V_{\uparrow \downarrow}$ is proportional to $P_{G a A s}$, where $P_{G a A s} \equiv$ $\left(n_{\uparrow}-n_{\downarrow}\right) /\left(n_{\uparrow}+n_{\downarrow}\right)$ and $n_{\uparrow}$ and $n_{\downarrow}$ are the densities of up and down spins respectively. Under forward bias $P_{G a A s}$ increases with increasing $V_{i n j}$, as shown in Fig. 2 .

The other important aspects the data of Fig. 1(b) are the depolarization $\operatorname{dip}\left(V_{N L} \approx\right.$ $\left.V_{\uparrow \downarrow}\right) / 2$ and re-polarization peak $\left(V_{N L}=V_{\uparrow \uparrow}\right)$ at low field $\left(\left|B_{x}\right|<150\right.$ Oe). These features are shown on an expanded scale for several interfacial bias voltages in Figs. 3)(a) and (b). A three-terminal measurement ${ }^{\underline{1}}$ is sensitive only to accumulation of spins parallel to the magnetization of the injector itself and hence is a direct probe of spin precession in the semiconductor. The fact that the field dependence of the two types of measurements are 
very similar indicates that the low-field signatures are due entirely to precession. It can be easily verified, however, that the effective field leading to the precessional dynamics is much larger than the applied field. This reflects the existence of a hyperfine field due to dynamically polarized nuclei, which has the following form: ${ }^{10}$

$$
\vec{B}_{N}=b_{n} \frac{\left(\vec{B}+b_{e} \vec{S}\right) \cdot \vec{S}\left(\vec{B}+b_{e} \vec{S}\right)}{\left(\vec{B}+b_{e} \vec{S}\right)^{2}+\xi B_{l}^{2},}
$$

where $b_{n}$ and $b_{e}$, which are both negative in GaAs, $\stackrel{10}{=}$ represent effective fields due to the polarized nuclei and electrons, $\vec{S}$ is the average electron spin $\left(|\vec{S}|=1 / 2\right.$ for $\left.P_{G a A s}=100 \%\right)$, $B_{l}$ is the local dipolar field experienced by the nuclei, and $\xi$ parameterizes the assisting processes which allow energy to be conserved in mutual spin flips between electrons and nuclei. ${ }^{14}\left|\vec{B}_{N}\right|$ can be as large as several Tesla in our samples.

From Eq. 1, we determine that at large $B_{x}, \vec{B}_{N}$ is essentially anti-aligned with $\vec{S}$, as shown in Fig. 3(c), and $\vec{S}$ remains polarized, with negligible dephasing from precession. However, when $B_{x}$ becomes comparable to $B_{z}, \vec{B}_{N}$ rotates towards the $z$-axis, as shown in Fig. $3\left(\right.$ d). $\vec{S}$ precesses around $B_{N, z}$ resulting in the observed depolarization. At a smaller field $B_{r}$, indicated by the arrow in Fig. 3(a), the electron spin system becomes re-polarized, indicating that the nuclear field has been suppressed. This phenomenon has previously been associated with the cancellation of the magnetic field along the spin injection axes $B_{x}$ by the electronic exchange field $b_{e} \vec{S}$ acting on the nuclei. $\frac{15}{}$ When $B_{x}$ is swept from the opposite direction the spin polarization $\vec{S}$ and hence the Knight field are opposite in sign, and the sign of $B_{r}$ reverses, as shown in Fig. 11(b).

In optical orientation experiments, the argument of the previous paragraph has been used to determine $b_{e}$ by setting $B_{r}=b_{e} S$, where $S$ is the optically generated spin accumulation. $\stackrel{10,15}{15}$ In our experiment, we can enhance $S$ by increasing the injector bias. As shown in Fig. 2(inset), however, $B_{r}$ as measured by either the non-local or three-terminal methods clearly decreases with increasing bias, in direct contradiction of the bias dependence of $V_{\uparrow \uparrow}-V_{\uparrow \downarrow}$ shown in Fig. 2, This anomaly, which precludes a simple identification of the Knight field, is due to the fact that the electron spin polarization at small fields is reduced by precession. This fact, which was ignored in the interpretation of the early optical orientation measurements, has a profound influence on the electron spin dynamics at very low fields.

To address this shortcoming as well as other aspects of our experiments, including the 


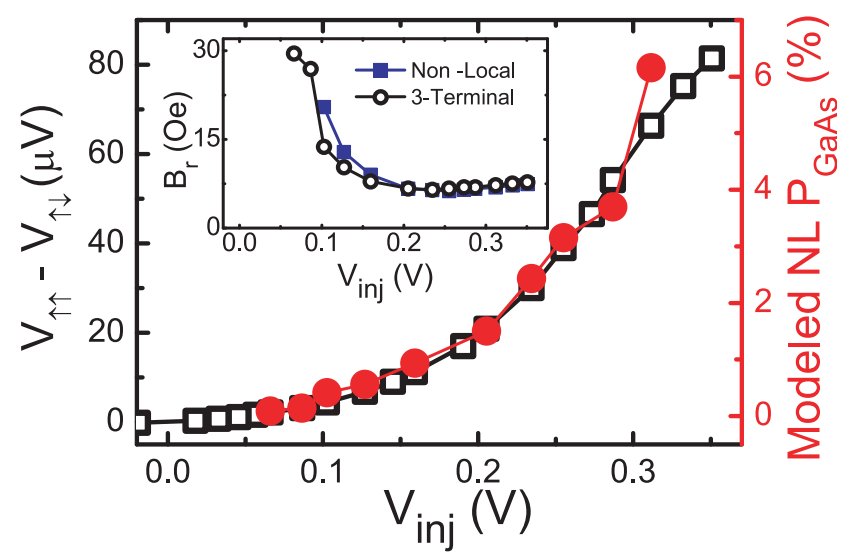

FIG. 2: (Color online) $V_{\uparrow \uparrow}-V_{\uparrow \downarrow}$ (black open squares, left axis) and modeled $P_{G a A s}$, the spin polarization averaged over the area of the non-local detector, i.e. under contact $c$, (red circles, right axis) as a function of injector bias. Inset: re-polarization field $B_{r}$, as defined in the text, for non-local (blue solid squares) and 3-terminal (black open circles) measurements as a function of injector bias.

spatial inhomogeneity in both the electron and nuclear spin polarizations, we consider a more complete model based on the drift-diffusion formalism that has been applied widely to spin transport problems. $\underline{1}_{2}^{2}, 11,13,16$ The dynamics of the injected spins in the GaAs channel are given by the following rate equation:

$$
\frac{\partial \vec{S}}{\partial t}=-v_{d} \frac{\partial \vec{S}}{\partial y}+D \frac{\partial^{2} \vec{S}}{\partial y^{2}}-\frac{\vec{S}}{\tau_{s}}-\vec{\Omega}_{L} \times \vec{S},
$$

where $v_{d}, D$, and $\tau_{s}$ are the drift velocity, diffusion constant, and spin lifetime; $\Omega_{L}=$ $g \mu_{B} \vec{B}_{t o t} t / \hbar$ is the Larmor frequency, $g=-0.44$ is the electron g-factor in GaAs, $\mu_{B}$ is the Bohr magneton, and $\vec{B}_{t o t}=\vec{B}+\vec{B}_{N}$ is the sum of the external applied field and the hyperfine field defined in Eq. 1. In order to model the experimental data, we solve Eq. 2 using the Crank-Nicholson method 17 with a one-dimensional spatial grid. A constant spin generation rate $\dot{S}_{0}$ is introduced in each cell beneath the injector. $\vec{S}$ and $\vec{B}_{N}$ are calculated at each position for each time step, thus leading to a self-consistent steady-state solution (typically after $50 \mathrm{~ns}) \stackrel{18}{\underline{18}}$ The non-local and 3-terminal curves as a function of $B_{x}$ are determined from the spatial average of $S_{x}$ at the detector and injector respectively. The parameters $v_{d}$ and $D$ are determined independently from Hall and resistivity measurements. In the simulations, we set $v_{d}=0$ outside of the charge current path, i.e. between the injector and detector. $b_{n}, b_{e}, \sqrt{\xi} B_{l}$, and $\tau_{s}$ are obtained from fits of a field sweep at one bias current and are then 


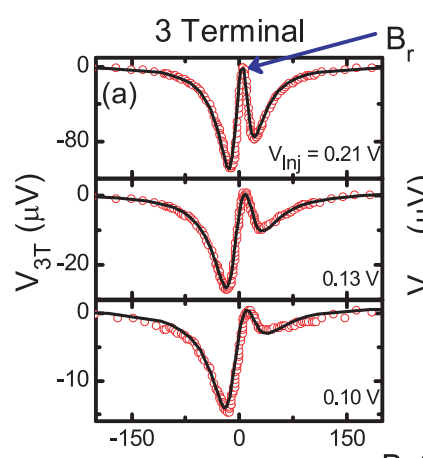

(c)
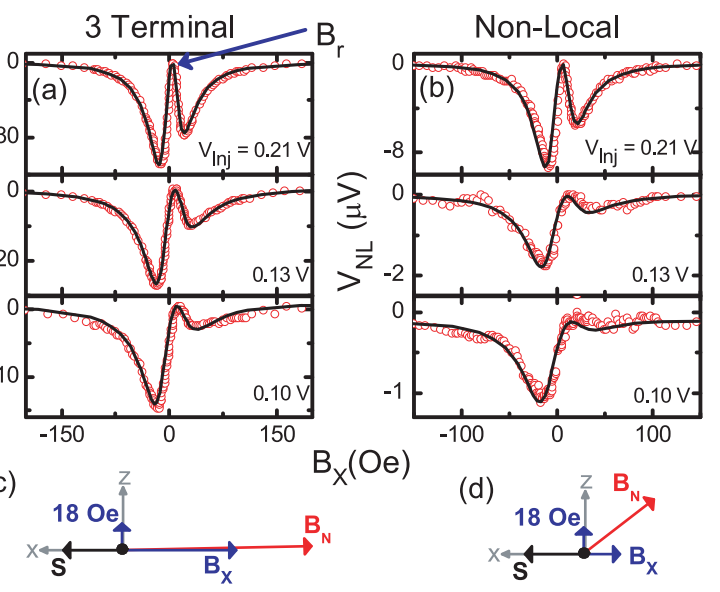

(d)

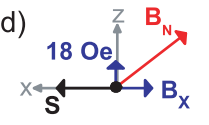

FIG. 3: (Color online)3-terminal voltage $V_{3 T}$ (a) and non-local voltage $V_{N L}$ (b) as a function of applied field $B_{x}$ in the measurement configuration shown in Fig. 1(a) for different injector biases $V_{i n j}$. Open circles are experimental data for $B_{x}$ swept from large negative field at $T=60 \mathrm{~K}$. Solid lines are modeling results as described in the text. Schematics showing the direction of the hyperfine nuclear field $\vec{B}_{N}$ for large $B_{x}$ (c), and for $B_{x}$ comparable to the transverse field $B_{z}(\mathrm{~d})$.

kept fixed while fitting the data for other bias currents. Only $\dot{S}_{0}$ varies with bias, but it is kept the same for simulations of the same bias. As indicated by the solid curves in Figs. 3(a) and (b), the modeling clearly reproduces the measured curves and the re-polarization fields. 19

From the modeling we determine that $b_{n}=-53 \mathrm{kOe}$ and $b_{e}=-50$ Oe, which are, as expected, smaller than the theoretical ideal values of $b_{n}=-170 \mathrm{kOe}$ and $b_{e}=-170$ Oe (calculated by Paget et al. $\underline{\underline{10}}$ for a closed electron-nuclear spin system and in which the donor sites are always occupied by spin-polarized electrons) but larger by a factor of 1.3 to 4 then previously measured values for $p$-type GaAs ${ }^{10}$ and Ge-doped GaAs. ${ }^{15}$ We find $\sqrt{\xi} B_{l}=40$ Oe. $\xi$ incorporates sample specific processes that assist nuclear spin polarization and is given by $\xi=T_{p o l} / T_{1}\left(B / B_{l}\right)^{2} . \underline{10}$ Using $T_{p o l} \approx 10 \mathrm{~s}, \stackrel{20}{\underline{n}} B_{l}=1.45$ Oe, $\stackrel{10}{\underline{10}}$ and a measured $T_{1}=40 \mathrm{~s}$ at $B=100$ Oe, we find $\sqrt{\xi} B_{l}=50$ Oe, which is comparable to our measured value.

The complete consistency among the different curves in Fig. 3 given a single set of parameters allows us to use this approach to measure the bias dependence of the spin polarization $P_{G a A s}=2\langle|\vec{S}|\rangle$ averaged over the non-local detector. The results extracted from the modeling, shown in Fig. 2, are in excellent qualitative agreement with the bias dependence of $V_{\uparrow \uparrow}-V_{\uparrow \downarrow}$. It is extremely important to note that this measurement of $P_{G a A s}$, which is based 

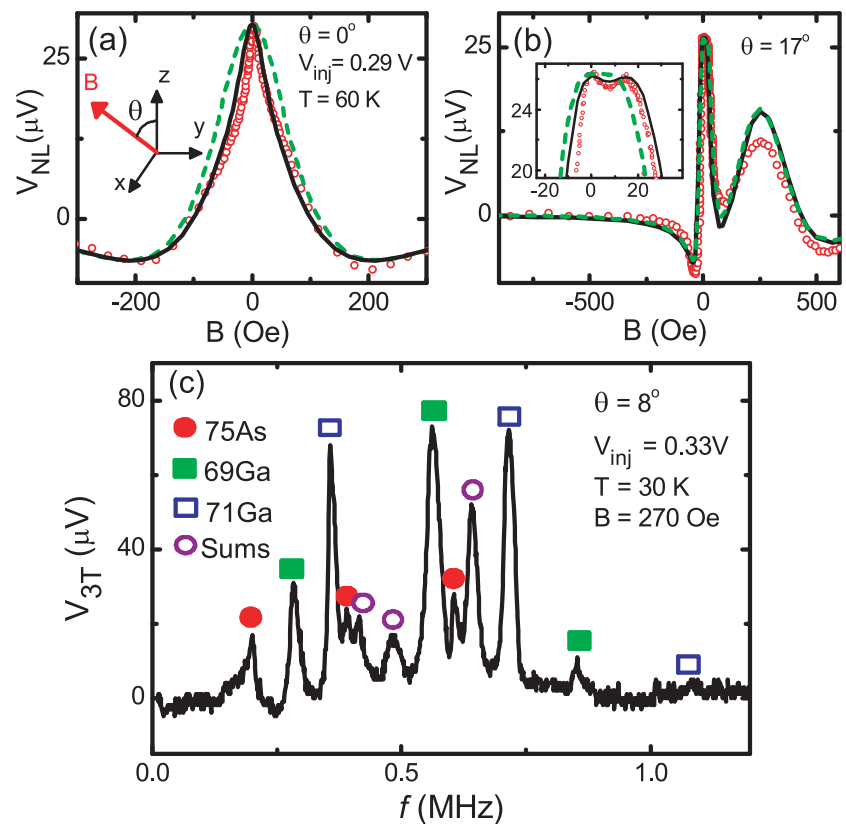

FIG. 4: (Color online) Non-local voltage field dependence: measured curves (red circles)and modeled curves with Knight field (black line) and without Knight field $\left(b_{e}=0\right.$, green dashed line) for magnetic field swept along the $\mathrm{z}$ axes (Hanle) (a) and along polar angle $\theta=17^{\circ}$ from the $\mathrm{z}$ axes (oblique Hanle) (b). Modeled curves are calculated with the same parameters used to fit data in Fig. 3. (b) Inset: closeup of the oblique Hanle curves. (c) The three-terminal voltage $V_{3 T}$ as a function of frequency of applied ac magnetic field. The observed resonances for each isotope are indicated in the legend. Transitions at the sums of the fundamental frequencies of different isotopes are also observed.

on the Knight field, is independent of any of the usual assumptions underlying spin transport measurements. It is therefore of particular interest to compare, quantitatively, with the polarization as inferred from the non-local voltage using standard arguments. Following Ref.13, $P_{G a A s}$ can be estimated from $V_{\uparrow \uparrow}-V_{\uparrow \downarrow}=2 \eta P_{F e} P_{G a A s} E_{f} / 3 e$, where $\eta$ is the interface spin transmission efficiency, $P_{F e}=0.4^{21}$ is the spin polarization of Fe, and $E_{f}$ is the fermi energy of GaAs, which is assumed to be the that of a Pauli metal with an effective mass $m^{*}=0.07 m_{e}$. From the measured $V_{\uparrow \uparrow}-V_{\uparrow \downarrow}$ in Fig. 2 we have calculated $P_{G a A s}$ (for $\eta=0.5$, which is expected based on spin-LED measurements ${ }^{22}$ and a measured carrier density of $4.3 \times 10^{16} \mathrm{~cm}^{-3}$ at $\left.T=60 \mathrm{~K}\right)$ to be between $0.2 \%$ and $6 \%$ for the injector bias range shown. This is in good agreement with the values obtained from the analysis of the coupled electron-nuclear spin dynamics shown in Fig. 2. It is not obvious a priori that this should 
be the case, since the spin-polarized electrons responsible for the Knight shift are bound on donors while those probed by the non-local measurement are at the Fermi level. Among the important implications of this result is that the magnitude of the density of states in the semiconductor near the $\mathrm{Fe} / \mathrm{GaAs}$ interface is not grossly different from that given by the naive Pauli model. We have also implemented this analysis on a separate Fe/GaAs heterostructure with the same channel doping but with $V_{\uparrow \uparrow}-V_{\uparrow \downarrow}$ an order of magnitude lower. Fits with the same parameters as those used to model data in Fig. 3 yielded $P_{G a A s}$ that are in agreement with the significantly lower spin signal.

The extraordinary sensitivity of the electron spin dynamics to hyperfine effects in the FS system was initially observed in the distortion of the Hanle effect, in which electron spin polarization is suppressed by precession in a transverse magnetic field. $\stackrel{1}{*}$ Fig. (4) shows $V_{N L}$ for transverse field swept along the $z$-axis and at a polar angle of $17^{\circ}$ from the $z$-axis (the static field in Fig. 1(a) is eliminated). The salient features of these data are reproduced by the model introduced above with exactly the same parameters used to fit the data of Fig. 3. Of particular interest are the narrowing of the Hanle curve in Fig. 4(a) and the weak splitting of the zero-field peak shown in the inset of Fig. 4(b). Both are due to the effect of the Knight field. In its absence, the Hanle curves in Fig. 4(a) and (b) would show the form indicated by the dashed curves. We believe that the model's overestimation of the magnitude of the high-field satellite peak in Fig. 4(b) is due to the assumptions of purely one-dimensional diffusion as well as a uniform current density across the injection contact.

A final indication of the strong coupling of the electron and nuclear spin systems is provided by the detection of nuclear magnetic resonance (NMR) when an ac magnetic field is applied by a small coil placed over the sample. This is illustrated in Fig. 4 (c), in which $V_{3 T}$ is shown as a function of frequency in a constant oblique magnetic field at $T=30 \mathrm{~K}$. When the nuclei are off-resonance, the electron spin polarization is suppressed by precession around $B_{N}$. At a resonance, the nuclei are partially thermalized, $B_{N}$ is reduced, and the electron spin polarization is restored. At low fields, this approach can be used to observe all of the possible transitions in the $I=3 / 2$ manifold for each of the three isotopes $\left({ }^{69} \mathrm{Ga}\right.$, ${ }^{71} \mathrm{Ga}$, and $\left.{ }^{75} \mathrm{As}\right)$ present in the sample. The observation of higher order $(\Delta m= \pm 2, \pm 3)$ transitions is likely due to dipolar interactions (which also lead to the "sum transitions" from two different isotopes) or quadrupolar coupling. $\underline{\underline{23}}^{2}$

In conclusion,we have demonstrated that the coupled electron-nuclear spin dynamics in 
$\mathrm{Fe} / n$-GaAs spin transport devices can be understood quantitatively, providing an independent means of determining the electron spin polarization. This work was supported by NSF under DMR 0804244, the ONR MURI program, the NSF NNIN program, and the Japan Society for the Promotion of Science (T.K.).

* On leave from Tokyo Institute of Technology, Yokohama, Kanagawa 226-8503, Japan. Current address: Corporate R\&D center, Toshiba Corp., Kawasaki, Japan

1 X. Lou, C. Adelmann, M. Furis, S.A. Crooker, C.J. Palmstrøm, and P.A. Crowell, Phys. Rev. Lett. 96, 176603 (2006).

2 X. Lou, C. Adelmann, S.A. Crooker, E.S. Garlid, J. Zhang, K.S.M. Reddy, S.D. Flexner, C.J. Palmstrøm, and P.A. Crowell, Nat. Phys. 3, 197 (2007).

3 I. Appelbaum, B. Huang, and D. Monsma, Nature (London) 447, 295 (2007).

4 M. Tran, H. Jaffrès, C. Deranlot, J. M. George, A. Fert, A. Miard, and A. Lemaître, Phys. Rev Lett. 102, $036601(2009)$.

5 C. Awo-Affouda, O.M.J. van 't Erve, G. Kioseoglou, A.T. Hanbicki, M. Holub, C.H. Li, and B.T. Jonker, Appl. Phys. Lett. 94, 102511 (2009)

6 M. Ciorga, A. Einwanger, U. Wurstbauer, D. Schuh, W. Wegscheider, and D. Weiss, Phys. Rev. B 79, $165321(2009)$.

7 G. Salis, A. Fuhrer, and S. F. Alvarado, arxiv:cond-mat/0908.0273

8 Optical Orientation, edited by F. Meier and B.P. Zakharchenya (North-Holland, New York, 1984).

9 A.W. Overhauser, Phys. Rev. 92, 411 (1953).

10 D. Paget, G. Lampel, B. Sapoval, and V.I. Safarov, Phys. Rev. B 15, 5780 (1977).

11 S.A. Crooker et. al., Science 309, 2191-2195 (2005).

12 A.T. Hanbicki et. al., Appl. Phys. Lett. 82, 4092-4094 (2003).

13 M. Johnson and R.H. Silsbee, Phys. Rev. Lett. 55,1790 (1985); Phys. Rev. B 37, 5326 (1988).

14 We adopt a notation similar to that used by Paget et al. in Ref.10, except that we have excluded additional numerical prefactors for $b_{n}$ and $b_{e}$ (the leakage factor $f$ and effective donor occupancy $\Gamma)$ which cannot be determined independently.

15 B.P. Zakharchenya, V.K. Kalevich, V.D. Kul'kov, and V.G. Flě̈sher, Fiz. Tverd. Tela 
(Leningrad) 23, 1387-1394 (1981).

16 F. J. Jedema, H. B. Heersche, A. T. Filip, J. J. A. Baselmans, and B. J. van Wees, Nature (London) 416, $713(2002)$

17 W.H. Press, S. A. Teukolsky, W.T. Vetterling, and B.P. Flannery Numerical Reciepes in C, The Art of Scientific Computing $2^{\text {nd }}$ ed.,(Cambridge University Press, 1992).

18 This approach ignores the dynamics of the nuclear spin system, for which Eq. 1 is true only in equilibrium. It will nevertheless arrive at a correct self-consistent steady-state solution for the coupled electron-nuclear spin dynamics, provided that the solution at equilibrium is unique.

19 In fitting the data, the error in $\dot{S}_{0}$ is estimated to be about $20 \%$. The error in nuclear field parameters is less than $20 \%$.

20 M.I. Dyakonov and V.I. Perel, in Optical Orientation, edited by F, Meier and B.P. Zakharchenya (North-Holland, New York, 1984)

21 R.J. Soulen Jr. et. al., Science 282, 85-88 (1998).

22 C. Adelmann, X. Lou, J. Strand, C.J. Palmstrom, and P.A. Crowell, Phys. Rev. B 71,121301(R) $(2005)$.

23 J. Strand, X. Lou, C. Adelmann, B.D. Schultz, A.F. Isakovic, C.J. Palmstrøm, and P.A. Crowell, Phys. Rev. B 72155308 (2005). 